\title{
Aerobic exercise in mental disorders: from basic mechanisms to treatment recommendations
}

\author{
Andrea Schmitt ${ }^{1}$. Daniela Reich-Erkelenz ${ }^{2} \cdot$ Alkomiet Hasan $^{1} \cdot$ Peter Falkai $^{1}$
}

Published online: 26 June 2019

○) Springer-Verlag GmbH Germany, part of Springer Nature 2019

During the last years, several lines of evidence support the notion that aerobic exercise has the potential to improve symptoms and outcomes in severe mental disorders. Patients with schizophrenia (SZ) and major depression (MDD) have increased morbidity and mortality. For example, in a 12-year follow-up study, Speerfock et al. [1] found evidence for severity of MDD and bipolar disorder as important correlates of long-term changes of arterial hypertension and obesity. Increasing physical activity by aerobic exercise programs may improve metabolic syndrome and cardiovascular risk factors in patients with mental disorders, thus improving mortality [2]. A new meta-analysis [3] reveals that SZ patients have lower vigorous physical activity, and this variable correlates with disease-specific factors of social functioning such as withdrawal, low independence performance, employment and motivation/energy. For a long time, specific mechanisms of aerobic exercise-mediating effects on the pathophysiology of mental disorders were unknown. In a comprehensive review, Maurus et al. [4] summarize the effects of aerobic exercise on symptoms and cognition, global functioning and quality of life in SZ patients. Improvements in brain volumes and connectivity with special impact on those regions, which are affected in SZ, have been detected in previous studies. On the molecular level, the hypothalamus-pituitary-adrenal axis, growth factors, immune-related mechanisms, neurotransmitters and the endocannabinoid system may mediate the effects of aerobic exercise on brain plasticity.

In a randomized controlled study in patients with MDD, Buschert et al. [5] showed improved reaction time and shortterm verbal memory after a 4-week-lasting aerobic exercise

Andrea Schmitt

Andrea.Schmitt@med.uni-muenchen.de

1 Department of Psychiatry and Psychotherapy, University Hospital, LMU Munich, Munich, Germany

2 Institute of Psychiatric Phenomics and Genomics (IPPG), University Hospital, LMU Munich, Munich, Germany program. In this patient group and in patients with SZ [6], physical exercise is a feasible and easy-to-implement add-on therapy. The field of sports psychiatry, which is described by Ströhle [7], deals not only with side-effects of intense physical training in athletes, but also with aerobic exercise programs in patients with mental disorders. For some disorders such as MDD, mild cognitive impairment, dementia and post-traumatic stress disorder, complementary treatment with aerobic exercise has been included in current treatment guidelines on evidence level 1a. In SZ patients, the recent German treatment guideline recommends physical exercise with an evidence level B based on the Scottish Intercollegiate Guidelines Network (SIGN) classification. A recent meta-review of evidence and Position Statement from the European Psychiatric Association (EPA) [8] describes recommendations to fill existing research gaps and to increase the use of physical activity in routine clinical care in patients with severe mental illness. For instance, the amount of aerobic exercise, duration of treatment and combination with additional lifestyle interventions such as healthy diet and psychological programs have to be identified before aerobic exercise will be used widely in general practice. Petzold et al. [9] reveal that a structured psychological intervention is effective in outpatients with mental disorders to increase their level of physical activity in short- and mid term and to promote healthy diet. Finally, physical activity in an alpine outdoor environment has shown to improve resilience and quality of life beyond effects of physical activity itself in a large patient group with psychosomatic disorders, mostly somatoform disorder and MDD [10]. Ongoing studies investigate the impact of duration of aerobic exercise training. To maintain the effects of aerobic exercise beyond the training period, add-on treatment with drugs promoting neuroplastic processes in the brain has to be applied in future studies. 


\section{References}

1. Speerfock S, Dodoo-Schittko F, Brandstetter S, Apfelbacher C, Hapke U, Jacobi F, Grabe HJ, Baumeister SE, Schomerus G (2019) 12-year changes in cardiovascular risk factors in people with major depressive or bipolar disorder: a prospective cohort analysis in Germany. Eur Arch Psychiatry Clin Neurosci. https:// doi.org/10.1007/s00406-018-0923-1

2. Ohi K, Kataoka Y, Shimada T, Kuwata A, Okubo H, Kimura K, Yasuyama T, Uehara T, Kawasaki Y (2019) Meta-analysis of physical activity and effects of social function and quality of life on the physical activity in patients with schizophrenia. Eur Arch Psychiatry Clin Neurosci. https://doi.org/10.1007/s0040 6-018-0903-5

3. Schmitt A, Maurus I, Rossner MJ, Röh A, Lembeck M, von Wilmsdorff M, Takahashi S, Rauchmann B, Keeser D, Hasan A, Malchow B, Falkai P (2018) Effects of aerobic exercise on metabolic syndrome, cardiorespiratory fitness, and symptoms in schizophrenia include decreased mortality. Front Psychiatry 9:690

4. Maurus I, Hasan A, Röh A, Takahashi S, Rauchmann B, Keeser D, Malchow B, Schmitt A, Falkai P (2019) Neurobiological effects of aerobic exercise, with focus on patients with schizophrenia. Eur Arch Psychiatry Clin Neurosci. https://doi.org/10.1007/s0040 6-019-01025-w

5. Buschert V, Prochazka D, Bartl H, Diemer J, Malchow B, Zwanzger P, Brunnauer A (2019) Effects of physical activity on cognitive performance: a controlled clinical study in depressive patients. Eur Arch Psychiatry Clin Neurosci. https://doi. org/10.1007/s00406-018-0916-0
6. Ogunyankin F, Nichols H, Park S, Falkai P (2019) Effects of exercise-based interventions in severe mental illness: a feasibility study. Eur Arch Psychiatry Clin Neurosci. https://doi.org/10.1007/ s00406-018-0864-8

7. Ströhle A (2019) Sports psychiatry: mental health and mental disorders in athletes and exercise treatment of mental disorders. Eur Arch Psychiatry Clin Neurosci. https://doi.org/10.1007/s0040 6-018-0891-5

8. Stubbs B, Vancampfort D, Hallgren M, Firth J, Veronese N, Solmi M, Brand S, Cordes J, Malchow B, Gerber M, Schmitt A, Correll CU, De Hert M, Gaughran F, Schneider F, Kinnafick F, Falkai P, Möller HJ, Kahl KG (2018) EPA guidance on physical activity as a treatment for severe mental illness: a meta-review of the evidence and Position Statement from the European Psychiatrc Association (EPA), supported by the International Organization of Physical Therapists in Mental Health (IOPTMH). Eur Psychiatry 54:124-144

9. Petzold MB, Mumm JLM, Bischoff S, Große J, Plag J, Brand R, Ströhle A (2019) Increasing physical activity and healthy diet in outpatients with mental disorders: a randomized-controlled evaluation of two psychological interventions. Eur Arch Psychiatry Clin Neurosci 22:1-2. https://doi.org/10.1007/s00406-018-0941-z

10. Ower C, Kemmler G, Vill T, Martini C, Schmitt A, SpernerUnterweger B, Hüfner K (2019) The effect of physical activity in an alpine environment on quality of life is medicated by resilience in patients with psychosomatic disorders and healthy controls. Eur Arch Psychiatry Clin Neurosci. https://doi.org/10.1007/s0040 6-018-0930-2 